\title{
Research Staff Telecom Address
}

National Cancer Institute

\section{Source}

National Cancer Institute. Research Staff Telecom Address. NCI Thesaurus. Code

C94283.

A sequence of digits or characters used to identify a particular telephone, fax, or email of the research staff. 